Western University Scholarship@Western

2018

\title{
Mathematical Abilities in Children with Developmental Language Disorder
}

Alexandra M. Cross

Western University, across22@uwo.ca

Lisa M.D. Archibald

Western University, larchiba6@uwo.ca

Marc F. Joanisse

Western University, marcj@uwo.ca

Follow this and additional works at: https://ir.lib.uwo.ca/hrspub

Part of the Language and Literacy Education Commons, Rehabilitation and Therapy Commons, and the Science and Mathematics Education Commons

\section{Citation of this paper:}

Cross, Alexandra M.; Archibald, Lisa M.D.; and Joanisse, Marc F., "Mathematical Abilities in Children with Developmental Language Disorder" (2018). Health and Rehabilitation Sciences Publications. 14.

https://ir.lib.uwo.ca/hrspub/14 
Mathematical Abilities in Children with Developmental Language Disorder

\author{
Alexandra M. Cross ${ }^{1,2,3}$, Marc F. Joanisse ${ }^{2}$, \& Lisa M.D. Archibald ${ }^{1,2,3}$ \\ ${ }^{1}$ School of Communication Sciences and Disorders, The University of Western Ontario, \\ London, Canada \\ ${ }^{2}$ Brain and Mind Institute, The University of Western Ontario, London, Canada \\ ${ }^{3}$ Health and Rehabilitation Sciences, The University of Western Ontario, London, \\ Canada
}

Corresponding Author:

Alexandra M. Cross

Room 5175, Western Interdisciplinary Research Building, University of Western Ontario 1151 Richmond Street North

London, Ontario, Canada

N6A 5B7

Tel: 516-661-3529

Email:across22@uwo.ca

Conflict of Interest Statement: The authors have no conflicts of interest to disclose.

Funding Statement: No funding was received for this work. 


\begin{abstract}
Purpose. The present paper provides a scoping review of the literature on mathematical abilities in developmental language disorder (DLD). Children with DLD typically struggle with learning in school, however the mechanism by which DLD impacts academic success is unclear. Mathematics involves demands in the multiple domains, and therefore holds potential for examining the relationship between language and academic performance on tasks mediated by verbal and non-verbal demands.

Methods. A scoping review was performed via computerized database searching to examine literature on mathematics and DLD. The 21 papers meeting inclusion criteria compared children with typical development or DLD on various tasks measuring numerical cognition.
\end{abstract}

Results. Children with DLD consistently performed below peers with typical development on number transcoding, counting, arithmetic, and story problem tasks. However, performance was similar to peers with typical development on most number line, magnitude comparison, and conceptual mathematics tasks.

Conclusions. The findings suggest a relationship between DLD and mathematics was characterized by more detrimental performance on tasks with higher verbal demands. Results are discussed with respect to typical academic curricula and demonstrate a need for early identification and intervention in DLD to optimize academic outcomes.

Keywords: development language disorder, mathematics, numerical cognition, education 
Mathematical abilities in children with developmental language disorder

Successful learning in academic subjects is largely dependent on a child's ability to understand and use oral and written language, which places children with language impairments at risk for academic difficulties. Children with developmental language disorder (DLD), also known as specific language impairment, have impaired language abilities that are not associated with a known biomedical etiology (Bishop, Snowling, Thompson, \& Greenhalgh, 2017; Bishop, 1994). Children with DLD are at risk for learning difficulties in school, as DLD is likely to persist into school-aged years (Dockrell \& Lindsay, 2000; Stothard, Snowling, Bishop, Chipchase, \& Kaplan, 1998) and is known to impact academic outcomes (Beitchman, Wilson, Brownlie, Walters, \& Lancee, 1996; Dockrell, Lindsay, \& Palikara, 2011; Harrison, McLeod, Berthelsen, \& Walker, 2009). However, the mechanism by which language impairment impacts academic success is unclear. It is possible that these children struggle in school simply due to the verbal load of educational instruction and tasks. A second possibility is that differences associated with DLD extend beyond the verbal domain to impact other types of representations. An improved understanding of the link between DLD and academic success could lead to the development of better methods of early identification and intervention for educators and speech-language pathologists, which could, in turn, optimize long-term outcomes in children with DLD.

One area of education that has potential for elucidating the relationship between DLD and academic difficulties is mathematics. Studies have clearly demonstrated a link between early language difficulties and later development of mathematical abilities. Parent report on the Communication Domain of the Vineland Adaptive Behaviour Scales 
(Vineland; Sparrow, Balla, \& Cicchetti, 1984) of children's communication difficulties at 3 years of age predicts scores on the Key Math assessment of mathematical concepts and skills at 7 years old (Hall \& Segarra, 2007). Similarly, teacher reports demonstrate a link between early language abilities and later mathematics skills. In a longitudinal study of children with DLD, language abilities at 7 years old predicted teacher reports of students' mathematics performance at 11 years old (Durkin, Mok, \& Conti-Ramsden, 2015). These findings are also mirrored in behavioural results: studies of children with DLD have observed scores over one standard deviation below the population mean on standardized number tasks (Durkin, Mok, \& Conti-Ramsden, 2013) and achievement lower than the national distributions on curriculum assessments of mathematics (Conti-Ramsden, Knox, Botting, \& Simkin, 2002). Severity of language impairment at 7 years old predicted number skills one year later, and less improvement in language from 7 to 8 years old was associated with a drop in number skills performance. Overall, these studies clearly indicate a strong association between early language impairment and mathematical abilities in school-age years. However, the precise specification of this relationship remains unclear.

The relationship between DLD and mathematics is of particular interest as mathematics involves representations in both verbal and non-verbal domains, as described in the triple-code model of number processing (Dehaene, 1992; Dehaene \& Cohen, 1995). This model, illustrated in Figure 1, proposes that numerical tasks are supported by three types of mental representations or 'codes': the visual Arabic number form, in which numbers are represented as digit strings; the verbal word frame, which represents numbers in linguistic form; and the analogue magnitude representation frame, 
which consists of semantic representations of approximate quantity or magnitude. All numerical tasks are thought to involve accessing one or more code types or performing transformations from one code to another. Because there are multiple routes that link each code type to the two other code types, one can access two types of code without processing the third code. This is important because researchers can design tasks that aim to place high demands specifically on certain code types while minimizing demands on others, in order to examine different types of representations. With respect to language, studies can be designed to examine performance on tasks with a high verbal load compared to tasks that minimize verbal load and place demands on the visual or magnitude systems.

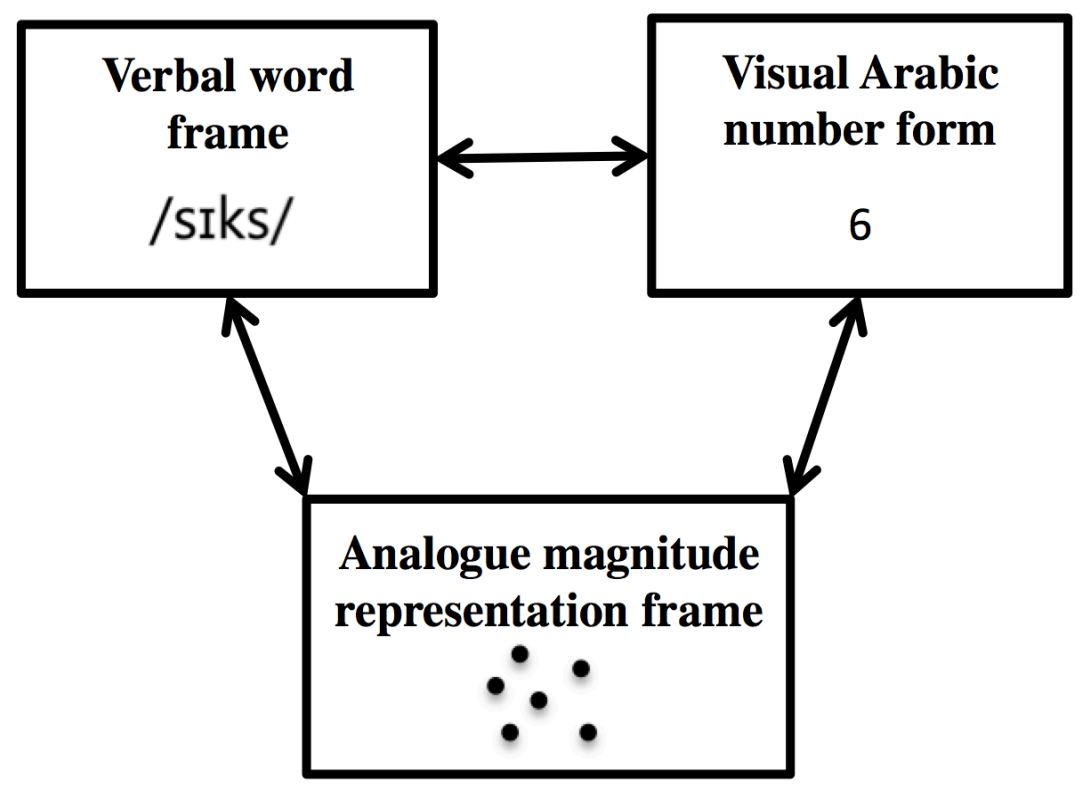

Figure 1. Illustration of the triple code model of number processing. Boxes represent code types and arrow represent transformations between code types. 
Measures typically used to assess mathematical abilities in children include number transcoding, counting, arithmetic, magnitude comparison, number lines, story problems, and conceptual tasks. The triple-code model posits that these tasks rely on different types of mental representations, as outlined in Table 1, allowing us to understand the different types of demands engaged by mathematics tasks. Number transcoding, counting, and arithmetic tasks are all thought to be tasks with significant demands in the verbal domain. Number transcoding, in which a participant names a written digit or writes the digit corresponding to a spoken number, involves transformations from visual Arabic number form representations to verbal word frame representations, and vice versa. Counting and arithmetic tasks rely on verbal word frame representations, although some variations of arithmetic tasks may also implicate the visual Arabic number form and analogue magnitude representation frame. In contrast, verbal demands are generally low in number line tasks, unless the task requires matching a spoken number to a number line, implicating verbal representations. Magnitude comparison tasks are typically used to assess symbolic and non-symbolic representations of magnitude, and are not expected to have a high verbal load. Within the triple-code model framework, the number comparison version of this task relies on the route from the visual form to the analogue magnitude representation, while the dot comparison task requires only magnitude representation given that no digits are present in the task.

Other commonly used mathematics tasks recruit different types of mental representations not included in the triple-code model. For example, in story problem tasks, mathematical problems are presented in an applied story format. While these tasks are likely supported by the same types of representations as an arithmetic task, they 
would also require integration of other types of phonological, morphosyntactic, and semantic knowledge in order for the child to understand and respond to the problem. Studies also commonly use conceptual tasks, which are designed to eliminate number words or digits in order to measure a child's underlying knowledge of a mathematics concept. The specific format of these tasks varies based on the type of conceptual knowledge being measured, but these are likely to rely on support from other types of representations beyond the triple-code model.

Table 1. Examples of mathematical tasks and the types of representations implicated in each task, based on the triple-code model of numerical cognition.

Type of Representation Implicated

\begin{tabular}{|c|c|c|c|c|}
\hline Task & $\begin{array}{l}\text { Verbal } \\
\text { Word } \\
\text { Frame }\end{array}$ & $\begin{array}{l}\text { Visual } \\
\text { Arabic } \\
\text { Number } \\
\text { Form }\end{array}$ & $\begin{array}{l}\text { Analogue } \\
\text { Magnitude } \\
\text { Representation } \\
\text { Frame }\end{array}$ & Examples \\
\hline $\begin{array}{l}\text { Number } \\
\text { Transcoding }\end{array}$ & + & + & & $\begin{array}{l}\text { Naming written numbers; writing } \\
\text { spoken numbers; matching } \\
\text { spoken and written numbers }\end{array}$ \\
\hline Counting & + & & & $\begin{array}{l}\text { Rote counting from 1; counting- } \\
\text { on from a higher number; } \\
\text { counting backwards; counting } \\
\text { objects }\end{array}$ \\
\hline Arithmetic & + & $++^{a}$ & & $\begin{array}{l}\text { Verbal recall of arithmetic facts; } \\
\text { written arithmetic problems }{ }^{a}\end{array}$ \\
\hline $\begin{array}{l}\text { Magnitude } \\
\text { Comparison }\end{array}$ & & $+{ }^{b}$ & + & $\begin{array}{l}\text { Comparing magnitude of digits } \\
\text { or dot arrays }\end{array}$ \\
\hline $\begin{array}{l}\text { Number Line } \\
\text { Task }\end{array}$ & $+^{c}$ & $+{ }^{d}$ & + & $\begin{array}{l}\text { Matching spoken }{ }^{\mathrm{c}} \text { or written }{ }^{\mathrm{d}} \\
\text { number to number line }\end{array}$ \\
\hline Story Problem & \multicolumn{3}{|c|}{$\begin{array}{l}\text { Depends on nature of problem, but } \\
\text { verbal demands are always high. }\end{array}$} & $\begin{array}{l}\text { Arithmetic problem presented in } \\
\text { applied story format }\end{array}$ \\
\hline Conceptual Task & \multicolumn{3}{|c|}{ Depends on nature of problem. } & $\begin{array}{l}\text { Includes tasks which assess } \\
\text { understanding of mathematical } \\
\text { principles, often using objects } \\
\text { instead of symbols. }\end{array}$ \\
\hline
\end{tabular}

Note: + notes significant demand on representations. Superscripts denote that demands are only significant when task is formatted as described in example with matching superscript. 


\section{How might DLD be related to mathematical abilities?}

Using the triple-code model, we can also make predictions about how mathematics performance might be expected to vary in children with DLD. The link between DLD and mathematics could theoretically occur in three forms, each of which would be manifested as a different pattern of performance on the tasks described above.

DLD may impact language broadly across multiple domains, which would create a barrier to learning in mathematics. This account is consistent with a domain-specific theory of DLD, in which the core deficit underlying DLD is specific to the verbal domain, including aspects of syntax (Rice, 2003; Van Der Lely, 2005; Wexler, Schütze, \& Rice, 1998) or phonology (Gathercole \& Baddeley, 1990; Joanisse \& Seidenberg, 2003). Under this account, problems with increased verbal demands would be particularly difficult for children with DLD. These children would be expected to struggle with number transcoding, counting, arithmetic, and story problems. However, children with DLD could also be able to acquire conceptual understanding of mathematics similar to typically developing (TD) peers when the linguistic load of instruction or assessment is reduced.

Alternately, language and mathematical cognition may be linked at the representational level, such that the same underlying factor would explain language and numerical processing in DLD. This would lead to specific deficits in representation of numbers and numerical relations in DLD. This account is consistent with domain-general theories of DLD, which propose a core deficit in a more general cognitive mechanism such as processing speed (Kail, 1991; Miller, Kail, Leonard, \& Tomblin, 2001), processing capacity (Leonard, 1998), symbolic processing (Stone \& Connell, 1993), or 
procedural memory (Ullman \& Pierpont, 2005). Under this account, language impairments in DLD are not merely a barrier to learning in a verbal format but also impact construction or processing of representations within other domains such as mathematics. Thus, difficulties would be expected to extend beyond the verbal domain to impact tasks relying on visual or magnitude representations such as number line and magnitude comparison tasks.

Finally, mathematical difficulties may simply be comorbid with language difficulties without sharing a common cause. Under this account, there is no causal relationship in which one impairment causes or influences the other; rather, there may be a third variable that causally explains both impairments. This differs from domain-general views of DLD as the two deficits simply co-occur without being causally related. This account would predict variable patterns of mathematics ability across children with DLD. Performance on mathematics tasks would vary independently of whether or not the child has DLD.

The present scoping review aims to examine mathematics performance in children with DLD in order to examine the first two accounts described above and to better understand the deficits underlying DLD. By reviewing performance on mathematics tasks in groups with and without DLD, we aim to examine whether impairments observed in DLD are verbally mediated or whether they also extend to nonverbal tasks. A scoping review was chosen here in order to broadly examine the extent, range, and nature of research activity in this area, and summarize relevant research findings (Arksey \& O’Malley, 2005). This differs from a systematic review in that the scoping review seeks to answer a broad rather than specific question, and presents a narrative account of research activity rather than synthesizing evidence. As a result, the present scoping 
review could lead to more focused primary research and systematic reviews that delve more narrowly into the subdomains we identified.

Specifically, we provide a scoping review of performance on mathematics tasks described above, including transcoding, counting, arithmetic, number lines, magnitude comparison, story problems, and conceptual tasks. A core deficit specific to the verbal domain is hypothesized to impact tasks with high verbal demands, including transcoding, counting, arithmetic, and tasks with a high linguistic load, such as story problems. Under this hypothesis, children with DLD should perform similarly to TD children on number line and magnitude comparison problems. Conversely, if differences in DLD extend beyond the verbal domain, children with DLD are expected to struggle on number line and magnitude comparison tasks. This paper did not explicitly aim to examine the hypothesis that DLD and mathematical abilities are comorbid but not causally related, as all the studies reviewed examined children with DLD as a group rather than differentiating between subgroups of children with DLD based on mathematical abilities. However, within the context of the scoping review below, this account would likely be manifested as a mixed pattern of results within specific mathematical tasks, based on differences in the proportion of children with DLD and children with DLD and comorbid mathematical difficulties selected in the sample.

\section{Methods}

To elucidate the relationship between language impairment and mathematics, a scoping review was conducted based on the scoping study framework outlined by Arksey and O’Malley (2005), including five stages: 1) identifying the research question; 2) 
identifying relevant studies; 3) study selection; 4) charting the data; and 5) collating, summarizing, and reporting the results.

\section{Phase 1: Identifying the Research Question}

The aim of the present scoping review was to summarize and disseminate research findings on mathematical abilities in children with DLD. Specifically, we aimed to answer the following question: How do school-aged children with DLD perform relative to TD peers on mathematical tasks with demands in verbal and non-verbal domains?

\section{Phase 2: Identifying Relevant Studies}

A comprehensive literature search for peer-reviewed articles was conducted using two computerized databases: PsycINFO and Web of Science. Broad search terms were used to generate a list of published journal articles using mathematics measures in children with DLD. These included "specific language impairment”, "primary language impairment", "language disorder", or "language disability"; and "math*”, "numer*”, "count*", "calculat*", "arithmetic", or "magnitude". The first four terms were chosen in order to capture the term "developmental language disorder" as well as alternate labels used to describe children with an impairment in language abilities with no known biomedical etiology (Bishop, 2014). The remaining terms related to mathematics were selected on the basis of terminology used in articles found in preliminary searches. A total of 255 studies were returned based on these search terms.

\section{Phase 3: Selecting Studies}

Following removal of duplicate papers, the titles and abstracts of the identified studies were first screened based on the following inclusion criteria. First, papers were 
required to include participants between the ages of 4 and 14 years old. This age range was selected to capture studies of school-aged children. Second, studies were required to include a group of participants identified with DLD by clinical professionals or by standardized assessment. DLD was defined as an impairment in language abilities with no known biomedical etiology. Studies using other labels were included in the present review when groups met criteria consistent with this definition of DLD. Children with DLD are heterogeneous on a range of variables including type of language impairment (expressive vs. receptive and expressive, pragmatic vs. morphosyntactic), written language abilities, nonverbal reasoning abilities, home environment, and instructional opportunities. The present scoping review did not exclude studies with heterogeneous participant groups, consistent with Bishop and colleagues' consensus study of terminology and definitions for DLD $(2016 ; 2017)$. Third, the studies were required to include at least one behavioural measure of mathematics, assessing some form of numerical representation. Such measures could include, but were not limited to, number transcoding, counting, arithmetic, number line tasks, magnitude comparison, story problems tasks, or tasks measuring conceptual understanding of mathematics. Fourth, the studies were required to include a comparison of children with DLD to an age-matched TD group or to published population norms on the aforementioned mathematics task. Fifth, the studies were required to be written in English. All screened articles were published before mid-2017, at the time the review was completed.

Two reviewers completed the review of the titles and abstracts. At the time of the review, one reviewer (first author of the present paper) was a doctoral student in a speech-language pathology program and the second reviewer was completing a master's 
degree in psychology. The second reviewer was trained by the first reviewer on the key components and terminology of the study prior to beginning the review process. The reviewers conducted one initial reliability trial run in order to establish some consistency in the review process. Each reviewer screened the abstracts and titles of 10 papers, and then met to discuss the exclusion and inclusion criteria. Following this trial run, they each independently reviewed the titles and abstracts of the full sample of papers resulting in the exclusion of 179 articles at this step.

Following screening, the two reviewers completed the full-text review of the remaining 21 studies, based on the inclusion criteria described above. The two reviewers met to discuss coding or inclusion and exclusion for any studies for which a discrepancy had occurred $(n=3)$. Fourteen full-texts were determined to meet the inclusion criteria. In addition, reference lists of the selected articles as well as review papers returned by the search were used to identify additional papers that matched the inclusion criteria. The cited articles were first screened based on their titles and then reviewed based on the fulltext using the inclusion criteria described above. Six articles were identified via this method, yielding a total of 20 studies for inclusion in the present review. Figure 2 illustrates the scoping review process and reasons why articles were excluded from the study in each phase.

\section{Phase 4: Charting the Data}

Data from the selected studies were extrapolated and charted in an Excel spreadsheet as follows. First, author(s) and year of publication were recorded. Second, details on the participant group(s) were noted, including sample size, age, and language status. Third, the mathematical tasks used were categorized into the following categories: 
number transcoding, counting, arithmetic, story problems, number lines, digit comparison, non-symbolic comparison, and conceptual problems. Fourth, results with respect to performance of DLD group relative to TD group were recorded, as well as other notes about correlations between tasks and additional analyses completed in the study.

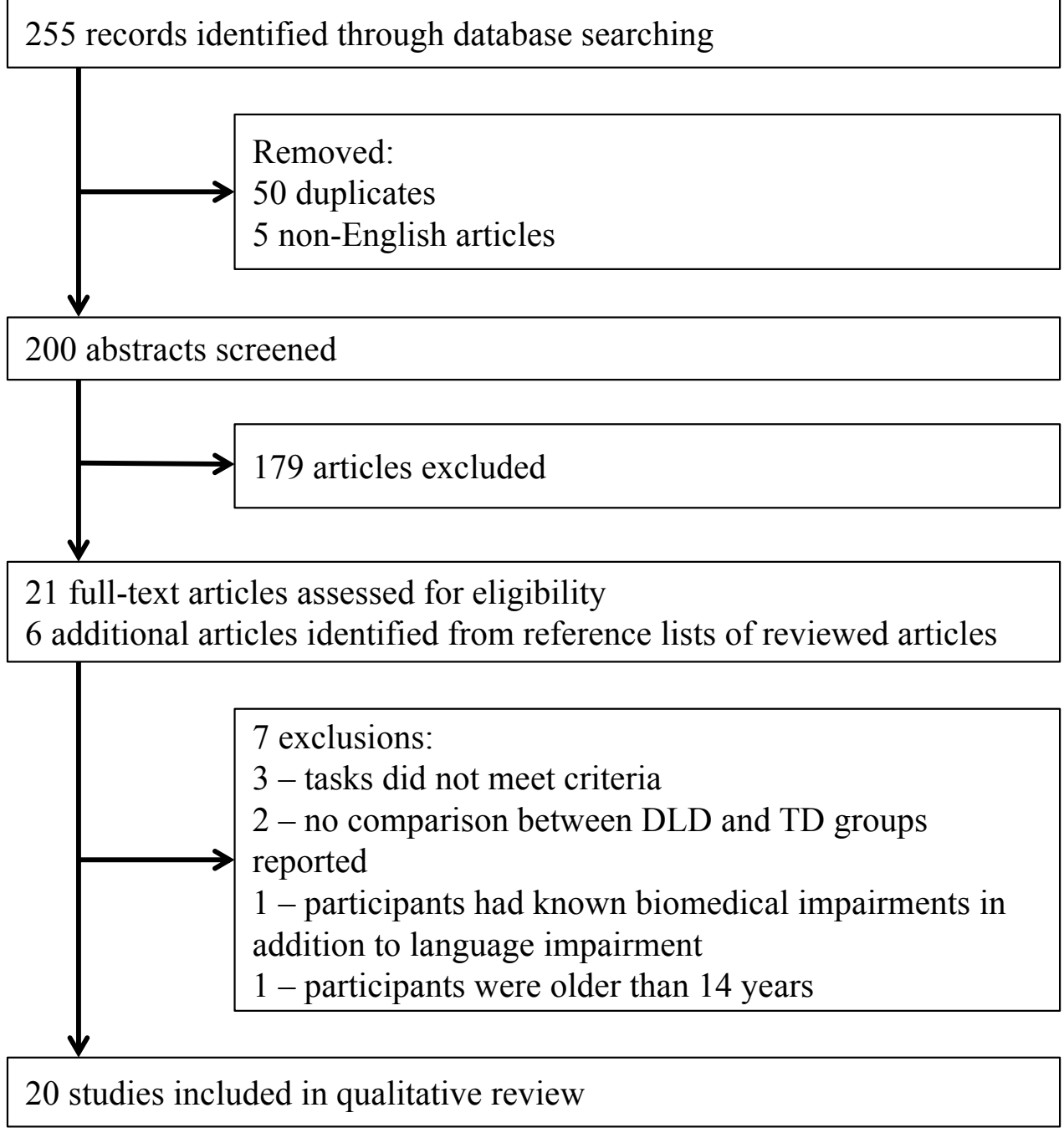

Figure 2. Flowchart illustrating the scoping review procedures. 


\section{Phase 5: Collating, Summarizing, and Reporting the Results}

After extracting the data, percentages of studies assessing each type of mathematical task were calculated. Qualitative results of each study were summarized in tables.

\section{Results}

\section{Quantitative Findings}

Interrater Reliability. The kappa statistic was used to examine interrater reliability of the two reviewers during the title and abstract screening and full-text review phases of the scoping review. The interrater reliability for the screening phase was $\kappa=$ $.97(p<.001), 95 \% \mathrm{CI}(0.94,1.00)$. For the full-text review phase, interrater reliability was $\kappa=.96(p<.001), 95 \%$ CI $(0.90,1.00)$. This suggests that there was a high degree of consistency between the two reviewers in applying the exclusion and inclusion criteria.

Types of mathematical tasks. Data regarding the type of mathematical task used in each study was extracted during the final phase of the scoping review, and the percentage of studies utilizing each task type was calculated. The included studies utilized a total of eight types of mathematical tasks, with each study often involving more than one task type. The eight tasks across the studies included number transcoding $(10 \%$ of studies), counting (45\%), arithmetic (60\%), digit comparison (35\%), non-symbolic comparison $(15 \%)$, story problems $(15 \%)$, and conceptual tasks $(20 \%)$.

\section{Qualitative Findings}

Findings of the reviewed papers are summarized in Table 2. Qualitative descriptions of results are provided below, based on the tasks used in each study. 
Table 2. Summary of findings of studies comparing performance on mathematics tasks in children with DLD and TD peers.

\begin{tabular}{|c|c|c|c|c|c|c|c|c|c|c|c|c|}
\hline Study & $\begin{array}{c}n(\mathrm{TD} \\
\text { age- } \\
\text { matched) }\end{array}$ & $\begin{array}{c}n(\mathrm{TD} \\
\text { language- } \\
\text { matched) }\end{array}$ & $\begin{array}{c}n \\
\text { (DLD) }\end{array}$ & $\begin{array}{c}\text { Age } \\
\text { range } \\
\text { of DLD } \\
\text { group } \\
\text { (years) }\end{array}$ & 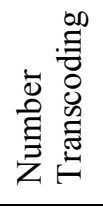 & 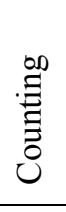 & 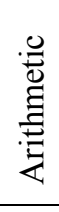 & 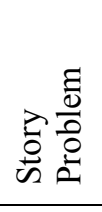 & 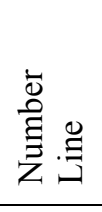 & 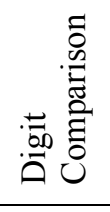 & 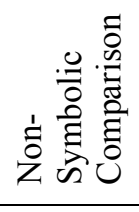 & $\begin{array}{l}0 \\
0 \\
0 \\
0\end{array}$ \\
\hline Nelson et al., 2011 & 116 & $\mathrm{n} / \mathrm{a}$ & 220 & 4 & & & & $\mathrm{X}$ & & & & \\
\hline Fazio, 1994 & 20 & 20 & 20 & $4-5$ & & $\mathrm{X}$ & & & & & & \\
\hline Arvedson, 2002 & 19 & 19 & 19 & $4-5$ & & $\mathrm{X}$ & & & & & & $=$ \\
\hline Willinger et al., 2017 & 61 & $\mathrm{n} / \mathrm{a}$ & 61 & $4-6$ & & & $\mathrm{X}$ & & & & & \\
\hline Jordan et al., 1995 & 33 & $\mathrm{n} / \mathrm{a}$ & 33 & $5-6$ & & & $\mathrm{X}$ & $\mathrm{X}$ & & & & $=$ \\
\hline Kleemans et al., 2011 & 111 & $\mathrm{n} / \mathrm{a}$ & 61 & $5-7$ & & $\mathrm{X}$ & & & $=$ & & & \\
\hline Donlan, 1993 & 17 & $\mathrm{n} / \mathrm{a}$ & 13 & $6-7$ & $\mathrm{X}$ & $\mathrm{X}$ & & & & $=$ & & \\
\hline Fazio, 1996 & 15 & 16 & 14 & $6-7$ & & $\mathrm{X}$ & $\mathrm{X}$ & & & & & \\
\hline Donlan et al., 1997 & $\mathrm{n} / \mathrm{a}$ & 37 & 12 & $6-7$ & & & & & & $=$ & $=$ & \\
\hline Kleemans et al., 2012 & 107 & $\mathrm{n} / \mathrm{a}$ & 53 & $6-8$ & & & $\mathrm{X}$ & & & & & \\
\hline Kleemans et al., 2013 & 100 & $\mathrm{n} / \mathrm{a}$ & 50 & $6-8$ & & & $\mathrm{X}$ & & & & & \\
\hline Alt et al., 2014 & 21 & $\mathrm{n} / \mathrm{a}$ & 20 & $6-9$ & & & & & & $\mathrm{X}$ & $=$ & \\
\hline Donlan \& Gourlay, 1999 & 13 & 12 & 13 & $7-8$ & & & & & & $=$ & $=$ & \\
\hline Cowan et al., 2005 & 57 & 55 & 55 & $7-9$ & $\mathrm{X}$ & $\mathrm{X}$ & $\mathrm{X}$ & $\mathrm{X}$ & & $\mathrm{X}$ & & \\
\hline Nys et al., 2013 & $\mathrm{n} / \mathrm{a}$ & $\mathrm{n} / \mathrm{a}$ & 28 & $7-14$ & & $\mathrm{X}$ & $\mathrm{X}$ & & & $\mathrm{X}$ & & \\
\hline Donlan et al., 2007 & 55 & 55 & 48 & 8 & & $\mathrm{X}$ & $\mathrm{X}$ & & & & & $=$ \\
\hline Alloway \& Stein, 2014 & $\mathrm{n} / \mathrm{a}$ & 50 & 40 & $8-10$ & & & $\mathrm{X}$ & & & & & \\
\hline Mainela-Arnold et al., 2011 & 17 & $\mathrm{n} / \mathrm{a}$ & 17 & $8-11$ & & & $\mathrm{X}$ & & & & & $\mathrm{X}$ \\
\hline Fazio, 1999 & 11 & 11 & 10 & $9-11$ & & $\mathrm{X}$ & $\mathrm{X}$ & & & & & \\
\hline Koponen et al., 2006 & 120 & 20 & 29 & $9-11$ & & & $\mathrm{X}$ & & & $\mathrm{X}$ & & \\
\hline
\end{tabular}

Note. $\mathrm{X}$ : abilities are significantly below same-age peers; $=:$ abilities are not significantly different from same-age peers 
Number Transcoding. Number transcoding tasks involve naming written numbers, writing spoken numbers, and matching spoken and written numbers. Only two studies to date have examined number transcoding in children with DLD, and both suggest that performance on this task is impaired relative to TD children. Cowan, Donlan, Newton, and Lloyd (2005) compared school-age children with DLD in mainstream schools, children with DLD in schools specialized for language disorders, TD agematched controls, and TD language-matched controls on all three types of transcoding tasks. Both groups with DLD transcoded numbers less accurately than the group of agematched controls. The children with DLD in special schools performed more poorly on transcoding tasks than the children with DLD in mainstream schools, and scored similarly to the language-matched control group. In a regression, the authors showed that oral language comprehension uniquely explained variance in transcoding skill, even when nonverbal reasoning and working memory ability were taken into account. This finding suggests that children with DLD have difficulty with number transcoding relative to TD children and that this is related to oral language abilities. In addition, Donlan (1993) demonstrated that this deficit is particularly pronounced on tasks with numbers of larger magnitudes. Children with DLD read and wrote numbers 1-9 as accurately as TD children, but struggled to perform the same task with double-digit numbers between 10 and 100. Overall, these findings demonstrate that children with DLD perform below the level of TD peers on transcoding problems, particularly those with numbers greater than 10.

Counting. Research in young children with DLD has demonstrated differences in both counting range and counting accuracy on a variety of tasks. Children with DLD 
have a lower count range than age-matched TD controls (Arvedson, 2002; Nys, Content, Leybaert, \& Oetting, 2013). Similarly, a series of longitudinal studies demonstrated that preschool children with DLD struggle to remember the count sequence, and go on to have delays in rote counting in later school-age years despite adequate conceptual number knowledge (Fazio, 1994, 1996, 1999). Results from Donlan (1993) suggested that counting larger, more complex quantities is of particular difficulty for children with DLD. Six-year-olds with DLD counted arrays of 2-6 dots as accurately as TD children, but scored lower than the TD group on a task requiring counting of complex sets of 6-9 items. Children with DLD also had difficulty manipulating the counting sequence by counting-on from a given number or by counting backwards (Donlan, Cowan, Newton, \& Lloyd, 2007; Nys et al., 2013).

Other studies have provided insight into the relationship between performance on counting tasks and other cognitive processes in children with DLD. Kleemans, Segers, and Verhoeven (2011) examined rote counting and counting of objects in 5- to 7-year-old children with and without DLD. Children with DLD performed more poorly on forward and backward rote counting tasks as well tasks involving counting organized and disorganized quantities of objects. In a regression model, phonological awareness and grammatical comprehension accounted for individual differences in performance on counting tasks. Additionally, the regression model showed significant relationships between naming speed and the counting tasks in the DLD group only. The authors did not observe significant relationships between nonverbal early numeracy measures and verbal tasks such as naming speed, phonological awareness, and grammatical ability, and therefore suggest that the relationship between these skills and counting are related to the 
linguistic demands of the counting tasks. Similarly, Cowan and colleagues (2005) demonstrated a link between linguistic tasks and counting in 7- to 9-year-old children with and without DLD. Individual differences in language comprehension predicted counting performance, as did the central executive processes of working memory and nonverbal reasoning. Together, these studies suggest that in children with DLD, counting proficiency is associated with other verbally mediated skills such as phonological awareness, naming speed, and receptive language, as well as more general cognitive processes later in development.

Arithmetic. Numerous studies have provided evidence for arithmetic deficits and difficulty with math fact retrieval in children with DLD throughout the school-aged years. Research in 6- to 11- year-olds with DLD has documented poor written addition and subtraction relative to controls (Alloway \& Stein, 2014; Donlan et al., 2007; Kleemans, Segers, \& Verhoeven, 2012, 2013; Mainela-Arnold, Alibali, Ryan, \& Evans, 2011; Willinger et al., 2017), poor accuracy on spoken arithmetic problems (Jordan, Levine, \& Huttenlocher, 1995), and a strong correlation between arithmetic accuracy and fact retrieval of addition problems (Cowan et al., 2005). Accuracy on addition and subtraction tasks in children with DLD is also correlated with linguistic skills such as phonological awareness and grammatical ability, as well as general intelligence (Kleemans et al., 2012; Kleemans et al., 2013). Interestingly, Kleemans and colleagues (2012) also found that naming speed was a predictor of arithmetic accuracy in the DLD group only. The authors suggested that rapid naming tasks and fact retrieval during addition and subtraction tasks both rely on rapid retrieval from long-term memory. As a 
result, children with DLD may struggle with recall of linguistic representations, leading to difficulty using fact retrieval strategies while solving arithmetic problems.

A series of longitudinal studies also suggested that early counting difficulties are related to later arithmetic difficulties in children with DLD. Fazio (1994) demonstrated that preschool-age children with DLD had difficulty remembering and retrieving the count sequence. These difficulties in early numeracy skills likely contribute to the delays in counting larger numbers and difficulty remembering simple math facts observed in the same group of children at 6 to 8 years of age (Fazio, 1996). A final follow-up at 9 to 10 years of age suggested that early counting difficulties contribute to later calculation delays (Fazio, 1999). Children with and without DLD completed timed and untimed arithmetic problems with two-digit numbers while experimenters monitored for use of any immature counting strategies such as written tallies, counting aloud, or counting on fingers. Children with DLD used counting strategies significantly more frequently than age-matched controls and younger 8-year-old controls. Accuracy on timed arithmetic problems was higher in the age-matched control group compared to the DLD group, and in the younger control group compared to the DLD group. On a mathematics facts task, children with DLD also recalled math facts less accurately than age-matched controls. Overall, these longitudinal findings suggest that children with DLD have difficulty using more sophisticated fact retrieval strategies while completing arithmetic problems, and are forced to rely instead on less efficient counting strategies.

Interestingly, Fazio (1999) also observed an effect of timing on accuracy. When no timing constraints were present, children with DLD performed calculations with similar accuracy to the younger control group. In contrast, no improvement from timed to 
untimed arithmetic was observed in the age-matched and younger control groups. The authors suggested that children with DLD may have shown improvement on untimed arithmetic tasks because of additional time for number fact retrieval, and hypothesized that this effect was not observed in TD children because number fact retrieval has become largely automatic leading to more efficient processing.

Koponen, Mononen, Räsänen, and Ahonen (2006) also provided evidence for processing speed differences in DLD on arithmetic tasks. In their study, 9- to 11-yearolds with DLD completed single-digit arithmetic as accurately as education-matched controls. However, the speed of their calculations was significantly slower and they frequently used slower counting strategies rather than fact retrieval. These findings suggest that while older children with DLD may be able to catch up to same age peers in terms of accuracy on simple arithmetic, they are still impaired in terms of the speed at which they can perform these problems.

Finally, the arithmetic deficits described in DLD may be specific to exact symbolic calculation. Nys and colleagues (2013) differentiate between exact arithmetic tasks, in which children were asked to exactly solve addition problems, and approximate addition tasks, in which children estimated whether the approximate sum of two symbolic digit arrays or non-symbolic dot arrays were larger than a third digit or dot array. Sevento fourteen-year-old children with DLD performed exact addition problems less accurately than age-matched and vocabulary matched peers. On the approximate addition task, children with DLD did not differ from controls when performing approximate additions of non-symbolic quantities, but were less accurate than age-matched children with symbolic quantities. However, the difference observed in the symbolic condition 
disappeared when accounting for cognitive abilities such as verbal short-term memory, executive function, finger discrimination, and verbal counting abilities. The authors suggested that exact arithmetic skills are dependent on language, based on the required use of verbal number representations, whereas approximate arithmetic skills rely on magnitude representations and are therefore not affected by language impairment.

In summary, these studies provide compelling evidence for arithmetic deficits in children with DLD, which may be related to earlier counting difficulties. In school-age years, children with DLD use less mature counting strategies, and perform less accurately and more slowly than their TD peers when recalling arithmetic facts and performing arithmetic problems. In DLD groups but not TD groups, speed of processing appears to be an important factor in calculation performance. Some evidence suggests that children with DLD are not impaired on approximate arithmetic problems, particularly when these tasks are non-symbolic, as these tasks are supported by magnitude representations rather than verbal representations.

Magnitude Comparison. Magnitude comparison tasks include digit comparison and non-symbolic comparison, in which non-symbolic quantities such as dots are compared. Studies of digit comparison in children with DLD have yielded somewhat mixed findings. Some research has suggested that while digit comparison performance of children with DLD is better than expected given their language abilities, it is still below the expected level for their age or level of education. Koponen and colleagues (2006) found that children with DLD performed better than language-matched controls but more poorly than education-matched controls on non-verbal numerical tasks including digit comparison. However, this study collapsed scores across a number of non-verbal 
numerical cognition tasks for this result, which makes it difficult to draw specific conclusions about magnitude comparison ability alone. Cowan and colleagues (2005) documented similar results for a magnitude comparison task with written numbers ranging from two to five digits. Seven- to nine-year-old children with DLD performed these magnitude comparisons more accurately than language-matched controls but less accurately than age-matched controls. Tasks measuring nonverbal reasoning, visuospatial working memory, and central executive function all contributed unique variance to performance on the magnitude comparison task, suggesting performance on this task may be more related to non-verbal cognitive components than linguistic components. Similarly, Nys and colleagues (2013) demonstrated that although children with DLD performed digit comparisons less accurately than age-matched controls, this difference was abolished when accounting for scores on cognitive tasks including verbal short-term memory, executive function, finger discrimination, and verbal counting. Alt, Arizmendi, and Beal (2014) also observed reduced accuracy in children with DLD compared to TD controls. However, this study did not examine the relationship of accuracy on digit comparison tasks to other cognitive processes, and there were significant differences in nonverbal intelligence between the TD and DLD groups. Given the findings of Nys and colleagues (2013) and Cowan and colleagues (2005), it seems plausible that differences between groups may be related to nonlinguistic factors rather than language ability.

In contrast, other studies have demonstrated that children with DLD perform digit comparison tasks with similar accuracy as same-age peers. Donlan and Gourlay (1999) administered tasks comparing the magnitude of two spoken single-digit numbers and the magnitude of two spoken double-digit numbers. Seven- to eight-year-olds with DLD did 
not differ significantly from age-matched controls in their speed and accuracy of singledigit and double-digit comparisons. Similarly, Donlan, Bishop, and Hitch (1998) demonstrated that six to seven year olds with DLD completed magnitude comparisons of single digits with similar accuracy to TD peers. In fact, response time on digit comparison tasks was faster for the group with DLD relative to the TD group. A study by Donlan (1993) did not find a significant group difference between children with and without DLD on a comparison task with numbers between 1 and 100. Data did suggest that more children with DLD failed to show mastery of the tasks, operationalized as at least 9/11 correct judgments, however, the study's sample was small and groups were not perfectly matched for age and nonverbal ability.

Although fewer studies have examined non-symbolic comparison in children with DLD, these have provided more consistent findings of unimpaired performance. Children with DLD performed similarly to age-matched controls on block size comparison tasks (Donlan \& Gourlay, 1999). Similarly, accuracy and speed on dot comparison tasks have not been found to differ in children with DLD and age-matched groups (Alt et al., 2014; Nys et al., 2013). In fact, Donlan, Bishop, \& Hitch (1998) observed faster response times in children with DLD.

In summary, this body of research presents mixed findings about magnitude comparison abilities in children with DLD. All reviewed studies documented digit comparison performance that is at least better than that of language-matched control subjects, and differences typically disappear when non-linguistic cognitive measures such as working memory, short-term memory, executive function, and nonverbal intelligence 
are accounted for. Studies using non-symbolic magnitude comparison tasks have more consistently documented that accuracy and speed is not impaired in children with DLD.

Number Line. On number line tasks, participants locate a spoken or written number on a line representing a range of numbers. To date only two studies of children with DLD have utilized a number line task. One such study used a composite score of nonverbal magnitude representation measures, including digit comparison, matching numerals to quantity, and number line estimation, making it difficult to draw conclusions about number line tasks specifically. However, the study demonstrated that children with DLD performed better than language-matched controls and similarly to educationmatched controls on nonverbal mathematics tasks including a number line task (Koponen et al., 2006). Only Kleemans and colleagues (2011) provided clear findings with respect to number line tasks in children with DLD. This study demonstrated that 5- to 7-year-olds with DLD did not differ from same-age peers in accuracy on a number line task in which participants placed spoken numbers on a line. A hierarchical regression analysis demonstrated significant effects of general intelligence and visuospatial working memory on the number line task, suggesting this task is unrelated to linguistic ability. Thus, studies to date suggest that children with DLD are unimpaired on number line tasks, although further studies are warranted to confirm that these findings are robust and replicable.

Story Problems. Although few studies have documented story problem performance in children with DLD, all existing literature shows that children with DLD struggle with story problems. While TD children scored similarly on nonverbal numerical problems, spoken number fact problems, and story problems, children with low language 
abilities scored significantly less accurately on story problems and spoken number fact problems than on nonverbal conceptual arithmetic problems (Jordan et al., 1995). The authors suggested that although this group may have competent nonverbal calculation skills, the added linguistic component of story problems and spoken problems may contribute to poor task performance. Similarly, children categorized as having low language abilities performed more poorly on the Applied Problems subtest of the Woodcock-Johnson III: Tests of Cognitive Abilities (Woodcock, McGrew, \& Mather, 2001), a task that presents mathematical problems in applied story format (Nelson, Welsh, Trup, \& Greenberg, 2011). Specifically, the authors categorized participants into strong language delay, moderate language delay, mild language delay, low-average language status, and high language status, based on measures of grammar comprehension, sentence repetition, and vocabulary. Scores on the Applied Problems task increased systematically with each level of improved language status. Finally, Cowan and colleagues (2005) examined performance on addition and subtraction problems presented in spoken story format to children with DLD, age-matched controls, and language-matched controls. The children with DLD answered story problems less accurately than age controls and similarly to language controls. Performance on the story problem task was predicted by language comprehension abilities, demonstrating that receptive language skills contribute significantly to children's ability to understand and solve story problems. Overall, these findings indicate that, as expected, language ability is highly related to success in story problem tasks, and as a result, children with DLD struggle on these tasks. It is likely that the arithmetic difficulties described above play 
some role in their accuracy, however studies have yet to dissociate the unique roles of calculation deficits and receptive language deficits in performance on story problems.

Mathematical Concepts. Many concepts underlie mathematical performance, so there is large variability in the types of conceptual knowledge investigated in research. However, when conceptual knowledge is considered overall, studies suggest that reducing linguistic load allows children with DLD to demonstrate understanding of a variety of mathematical concepts.

Children with DLD perform similarly to TD children on conceptual arithmetic tasks. Donlan and colleagues (2007) examined children's understanding of arithmetic principles by asking participants to verify addition and subtraction problems with unfamiliar symbols. Children were presented with two equations, one of which had been marked as correct, and asked to verify the second equation (e.g., given $\varphi+\beta=\varpi$, verify $\varphi+\varpi=\beta)$. There was no significant group difference in performance on this task between the age-matched group and the DLD group, suggesting that children with and without DLD do not differ in their understanding of arithmetic principles. Similarly, Jordan and colleagues (1995) demonstrated equivalent performance in TD and lowlanguage groups on a non-verbal conceptual arithmetic task. Children were shown a set of disks, which were then covered, and a second set of discs were shown to the child and added to the first set under the cover such that the two sets were not in view. The child was asked to replicate the number of disks under the cover. A similar procedure measured conceptual understanding of subtraction, as well as a three-term measure combining both addition and subtraction problems. Children with low language skills 
performed similarly on this task to children with average language skills, suggesting they had an equally good understanding of nonverbal calculation concepts.

Arvedson (2002) used four tasks to examine performance on numerical problemsolving tasks without requiring counting aloud. In the Reproduction of Sets Task, children were presented a set of items and were asked to numerically reproduce the set. The Numerosity of Sets task required children to place a number of balls in an opaque box, and then press a lever to retrieve balls one at a time, indicating when they had retrieved all the balls. Children were scored based on whether they pressed the lever the correct number of times. The Number Relevant Transformation of Sets task used a similar procedure, in which the child placed a number of balls in the box. However, the researcher then added or removed a ball to the box, before the child retrieved all the balls by pressing the lever. In order to complete this task accurately, children must understand basic addition and subtraction principles. In the Number Irrelevant Transformation of Sets task, children were required to demonstrate understanding of conservation of number. The experimenter showed the child two plates with a set of stickers on each and asked the child to identify which had more stickers. Then, out of the child's view, the experimenter transformed the plates such that stickers were spaced differently or varied in number. Children were once again asked to identify which had more stickers. For all four tasks, the set size was varied such that children completed each measure with sets of $4,5,6$, and 7 items.

On all four tasks overall, children with DLD performed more accurately than grammar-matched controls and no group difference was observed between the DLD and age-matched groups. Interestingly, an interaction of set size and group was observed on 
the Reproduction of Sets, Numerosity of Sets, and Number Relevant Transformation of Sets tasks such that scores were lower in the DLD group than the age-matched group for larger set sizes only. No interaction was observed for the Number Irrelevant Transformation of Sets task. The authors suggested that this task was the least languagedependent, and children with DLD were therefore able to reliably demonstrate understanding of conservation of number. Overall, the authors argued that children with DLD are performing well above the expected level given their expressive grammar abilities, and that they are best able to demonstrate their competence when the linguistic demands of the task are minimized.

This study also demonstrated an interesting relationship between performance on the conceptual tasks and counting. Although children in the TD groups demonstrated a boost in accuracy when counting aloud during the conceptual tasks, the DLD group accuracy dropped markedly when counting aloud. The authors suggested that children with DLD may have used problem-solving strategies differently from their peers in the conceptual tasks, and the linguistic demands of counting hindered their use of other strategies.

While these studies have demonstrated comparable conceptual understanding in TD and DLD groups, Mainela-Arnold and colleagues (2011) suggested that DLD groups differ in their understanding of mathematical equivalence. Mathematical equivalence is the concept that the two sides of an equation are equal to one another. The authors examined understanding of this concept using a task in which participants filled in the blank space in single-digit problems such as $5+3+2=5+$ or 
$6+4+5=\ldots+5$. This study also differentiated between groups of children with DLD who showed deficits in expressive language only or in both expressive and receptive language. Children in both DLD groups performed equivalence problems less accurately than the TD children. However, analyses of expressive and receptive language skills did not reveal a clear pattern of how language abilities related to performance on the mathematical equivalence problems, and the authors suggested that language alone could not account for differences in understanding of mathematical equivalence. Additionally, it is difficult to differentiate whether the difference between groups was related to conceptual understanding or computational factors. The equivalence task in this study used digits, which may rely on verbal code representations. As described in this review, children with DLD appear to struggle with tasks involving verbal code representations, and experience significant difficulty on arithmetic tasks. It seems therefore plausible that impaired performance in the DLD group was related to computational factors and use of verbal code rather than conceptual understanding of mathematical equivalence.

In summary, although the concepts examined across these studies vary widely, many studies suggest that children with DLD can attain similar levels of conceptual understanding to TD peers. These groups can demonstrate their competence on conceptual tasks that minimize linguistic load.

\section{Discussion}

The present scoping review examined studies of mathematics ability in children with DLD to elucidate the relationship between language impairment and mathematics ability. Analyses were guided by the framework of the triple-code model (Dehaene, 1992; 
Dehaene \& Cohen, 1995) according to which tasks relying on verbal representations of number include number transcoding, counting, arithmetic, and story problems are considered to impose high verbal demands. In contrast, number line, magnitude comparison, and conceptual tasks tend to rely on non-verbal domains and visual or magnitude representations of number. Deficits were consistently observed in children with DLD relative to TD peers on number transcoding, counting, arithmetic, and story problem tasks. Conversely, performance was closer to the level of TD peers on number line, magnitude comparison, and conceptual tasks. Overall, this pattern of results suggests that mathematical impairments in DLD are specific to the verbal domain, impacting tasks with high verbal demands. The evidence as a whole suggests that impairments do not extend to non-verbally mediated tasks such as those relying on visual or magnitude representation, nor to conceptual tasks in which verbal demands are reduced.

Some of the reviewed studies also examined predictors of mathematics performance in children with DLD. On number line and magnitude comparison tasks, which are thought to place demands on non-verbal domains, performance was predicted by nonverbal cognitive abilities including visuospatial working memory and nonverbal intelligence. Overall, language comprehension was a unique predictor of performance on number transcoding, counting, and arithmetic tasks, likely related to the linguistic demands of these types of mathematics tasks. Naming speed also predicted counting and arithmetic performance in children with DLD, but not in TD peers. This suggests that fluent phonological processing plays a unique role in facilitating accurate counting and arithmetic in children with DLD. Finally, phonological awareness was a strong predictor of counting and arithmetic ability, although the underlying mechanism explaining this 
relationship is unclear. Phonological awareness may play a direct role in verbal coding, or be related to verbal representations of number only through its association with overall language proficiency. Further investigation is required to dissociate the relationships between phonological awareness, expressive and receptive language, and verbal representations of number. One potential avenue for examining this association is by comparing children with DLD, children with reading disability, and TD children. Children with reading disability have impairments in phonological awareness but generally have typical expressive and receptive language skills (Lyon et al., 2004). Thus, comparisons between DLD and reading disability may help to elucidate the independent contributions of language and phonological awareness in mathematics.

Interestingly, one study also demonstrated that children with DLD could perform approximate non-symbolic arithmetic as accurately as TD peers despite the impairment observed on exact symbolic arithmetic. Nys and colleagues (2013) suggested that this pattern of performance occurs because exact arithmetic processes but not approximate number processes are impaired in DLD. An alternate explanation, consistent with the present review, is that removing the symbolic aspect of the arithmetic task reduced the verbal demand of the task. The studies reviewed above suggest that children with DLD are impaired specifically on verbally mediated tasks, which would impact performance on exact arithmetic with digits but not on approximate arithmetic problems with dots. Only one study to date has examined approximate arithmetic in children with DLD. Further studies are warranted to examine whether the difference in performance is related to exact and approximate processes or to verbal and non-verbal demands. 
Given the recent changes in terminology and definitions of language impairment in children, it is important to note that all of the reviewed studies used the term "specific language impairment" when describing their samples and only included participants who scored within the average range on non-verbal intelligence measures or for whom parents reported no cognitive and academic concerns beyond language. Thus, although we use the terminology and definition of DLD identified by Bishop et al. $(2016 ; 2017)$, the findings of the present study also pertain to the narrower definition of specific language impairment. This also suggests that the pattern of impairments on verbal mathematics tasks that we observed is not related to groups differences in non-verbal cognition.

Given that the impairments documented in DLD are generally limited to tasks supported by verbal representations, the present review is more consistent with a domainspecific account of DLD. However, some domain-specific theories such as those arguing for a deficit to a specific aspect of morphosyntax would likely have difficulty accounting for the findings of this review. The studies reviewed provide evidence that children with DLD struggle with learning and retrieving number names and verbal arithmetic facts, and using verbal representations during arithmetic. Linguistic domain-specific theories generally suggest that the core deficit of DLD lies in aspects of syntax or morphology, and although this could contribute to some difficulty with learning of number words or understanding and responding to story problems, it is unlikely that the deficit would have such a widespread and long-lasting impact. Additionally, the timing effects observed in some studies on arithmetic tasks, in which children with DLD achieve the same level of accuracy as TD peers when timing constraints are removed, suggest that children with DLD may have intact verbal representations of numbers but are slowed in retrieving 
those representations. The results of the present review are more consistent with domainspecific theories positing a phonological deficit. In particular, the relationship of naming speed to counting and arithmetic accuracy in children with DLD is suggestive of a link between performance on these mathematics tasks and fluent phonological processing. However, the tasks used to date are not sufficient to differentiate whether deficits in counting and arithmetic are related to impaired phonological processing of verbal number representations or to poor encoding and retrieval of verbal number representations.

In contrast, the effect of DLD on mathematics described in this paper is more specific than would be expected based on some domain-general accounts. Accounts of DLD hypothesizing deficits in processing capacity, processing rate, symbolic processing, or procedural memory would generally predict that the impact of language impairment would extend to non-linguistic tasks such as number line and magnitude comparison tasks. This does not appear to be the case, as the present review did not observe consistent patterns of deficits in children with DLD on tasks relying on non-verbal domains.

In summary, the impairments observed in children with DLD are most consistent with domain-specific theories of DLD, in which DLD impacts performance on verbally mediated mathematical tasks. Poor mathematical performance in children with DLD may be related specifically to challenges representing and processing information in the verbal modality. Within typical mathematics curricula, this type of impairment would likely impact academic success in many areas. For example, the Grade 1-8 Ontario mathematics curriculum (Ontario Ministry of Education, 2005) includes five strands: number sense and numeration; measurement; geometry and spatial sense; patterning and algebra; and 
data management and probability. The potential impact of DLD on each of these strands is outlined in Table 3. While children with DLD are likely to show areas of relative strength in each of these strands on tasks where verbal demands are reduced, challenges with verbally mediated tasks are likely to impact performance on activities involving counting and arithmetic such as mathematical operations, measurement problems, algebraic calculations, and data management and probability procedures.

Table 3. Potential impact of DLD on performance in strands of Grade 1-8 Ontario mathematics curriculum.

\begin{tabular}{|c|c|c|}
\hline Strand & Examples of Tasks & Potential Impact \\
\hline $\begin{array}{l}\text { Number Sense } \\
\text { and Numeration }\end{array}$ & $\begin{array}{l}\text { Counting; mathematical } \\
\text { operations; understanding } \\
\text { of number and magnitude }\end{array}$ & $\begin{array}{l}\text { Typical conceptual understanding of } \\
\text { number and magnitude but impairment } \\
\text { on counting and operations }\end{array}$ \\
\hline Measurement & $\begin{array}{l}\text { Understanding } \\
\text { measurable attributes, } \\
\text { units of measurement, and } \\
\text { relationships among } \\
\text { measurement units }\end{array}$ & $\begin{array}{l}\text { Typical conceptual understanding of } \\
\text { measurement but some impairment on } \\
\text { measurement tasks related to counting } \\
\text { difficulties }\end{array}$ \\
\hline $\begin{array}{l}\text { Geometry and } \\
\text { Spatial Sense }\end{array}$ & $\begin{array}{l}\text { Recognizing shapes and } \\
\text { figures; understanding } \\
\text { geometric properties }\end{array}$ & $\begin{array}{l}\text { Performance similar to TD peers due to } \\
\text { visuospatial focus }\end{array}$ \\
\hline $\begin{array}{l}\text { Patterning and } \\
\text { Algebra }\end{array}$ & $\begin{array}{l}\text { Recognizing, describing, } \\
\text { and generalizing patterns; } \\
\text { representing patterns } \\
\text { algebraically }\end{array}$ & $\begin{array}{l}\text { Patterning performance similar to TD } \\
\text { peers on tasks with reduced verbal load, } \\
\text { some impairment with algebraic } \\
\text { calculations }\end{array}$ \\
\hline $\begin{array}{l}\text { Data } \\
\text { Management } \\
\text { and Probability }\end{array}$ & $\begin{array}{l}\text { Gathering, organizing, } \\
\text { and displaying data; } \\
\text { exploring probability } \\
\text { experiments and models }\end{array}$ & $\begin{array}{l}\text { Typical performance when organizing } \\
\text { and displaying data if verbal load is } \\
\text { reduced, some impairment in data } \\
\text { management and probability } \\
\text { procedures }\end{array}$ \\
\hline
\end{tabular}


It is also important to consider the impact of instructional format on learning in mathematics. Oral and written language are the primary method of instruction in educational settings. Children with DLD may struggle to learn new and esoteric vocabulary and understand complex language used to convey mathematical concepts, creating a barrier to learning in verbal instructional formats. The studies reviewed in the present paper did not address instructional factors; therefore, no specific conclusions can be drawn about the impact of DLD on learning when instruction is presented verbally versus non-verbally. However, the clear relationship observed between DLD and verbally-mediated mathematics tasks suggests that the linguistic load of instruction may contribute to difficulty learning, using, and understanding the academic language required to learn math skills and demonstrate competency in this domain.

Strategies that reduce the linguistic load of classroom activities may assist children with children with DLD to compensate for verbal difficulties in mathematics. Mathematics activities frequently involve verbally-demanding instruction techniques such as rote learning of arithmetic. Children with DLD may learn, or demonstrate their learning, more successfully when classroom activities incorporate multiple modalities or manipulatives to reduce verbal demands. Although few studies to date have examined the effectiveness of mathematics intervention in children with DLD, a recent pilot study provides promising evidence that mathematics instruction emphasizing visualization can improve numeracy skills in children with DLD (Mononen, Aunio, \& Koponen, 2014). In this study, kindergarten students with DLD completed the RightStart Mathematics curriculum (Cotter, 2001), delivered throughout one academic year in a small classroom setting. The program emphasizes visualization using manipulatives such as abacuses, 
tiles, cards with symbols representing numerical quantities, and cards with digits.

Students initially begin applying mathematical concepts using a concrete manipulative, such as tiles or an abacus, then using a semi-concrete manipulative, such as cards with dots or tally marks to represent a quantity, and finally using an abstract representation, such as cards with digits. Teachers are encouraged to emphasize understanding rather than rote learning and stimulate discussion with students about the concepts.

In Mononen et al.'s pilot study (2004), performance on mathematical tasks in children with DLD receiving intervention was compared to TD peers who received business-as-usual mathematics instruction. Pre-intervention, children with DLD had weaker early numeracy skills compared to TD peers. However, following the intervention, children with DLD showed significant improvement and performed similarly to TD peers on tasks measuring number transcoding, number comparison, and arithmetic following the intervention. The group with DLD continued to perform near age-level six months post-intervention on number comparison and arithmetic tasks, although number transcoding skills were weaker than age-matched controls. It remains unclear whether gains in mathematical performance post-intervention were related to improvements in verbal mathematical skills or whether the visual focus of the intervention allowed children to rely more on intact visual representations of number. However, this study provides encouraging findings suggesting that early numeracy skills in children with DLD can benefit when mathematics instruction emphasizes visualization and understanding of concepts, rather than rote learning. Additional research is warranted to further examine the effectiveness of these strategies for children with DLD in the classroom. 
In summary, the literature collectively demonstrates a need for awareness of the impact of DLD on learning in academic subjects beyond language arts. The present review indicates that children with DLD are likely to struggle in any mathematical tasks that place demands on the verbal domain. This includes skills such as number transcoding, counting, and arithmetic, which form the building blocks of later learning in mathematics education. An additional challenge is that language is the core medium of instruction, and DLD impacts children's ability to understand content presented in class and to demonstrate learning if required to do so using oral or written language. Mathematics instruction which reduces verbal demands and engages multiple modalities may promote better mathematics learning and improve long-term academic outcomes for all children with DLD. 


\section{Acknowledgements}

We thank Krystal Harris for her assistance in the review process, and Dr. Janis OramCardy and Dr. Daniel Ansari, both of whom provided insight and expertise that greatly assisted the research. 


\section{References}

Alloway, T. P., \& Stein, A. (2014). Investigating the link between cognitive skills and learning in non-comorbid samples of ADHD and SLI. International Journal of Educational Research, 64, 26-31. https://doi.org/10.1016/j.ijer.2013.10.003

Alt, M., Arizmendi, G. D., \& R. Beal, C. (2014). The Relationship Between Mathematics and Language: Academic Implications for Children With Specific Language Impairment and English Language Learners. Journal of Speech, Language, and Hearing Services in Schools, 45(July), 220-233. https://doi.org/10.1044/2014_LSHSS-13-0003

Arksey, H., \& O’Malley, L. (2005). Scoping studies: Towards a methodological framework. International Journal of Social Research Methodology: Theory and Practice. https://doi.org/10.1080/1364557032000119616

Arvedson, P. J. (2002). Young children with specific language impairment and their numerical cognition. Journal of Speech, Language, and Hearing Research, 45(5), 970-982. https://doi.org/10.1044/1092-4388(2002/079)

Beitchman, J. H., Wilson, B., Brownlie, E. B., Walters, H., \& Lancee, W. (1996). Longterm consistency in speech/language profiles: I. Developmental and academic outcomes. Journal of the American Academy of Child and Adolescent Psychiatry, 35(6), 804-814. https://doi.org/10.1097/00004583-199606000-00021

Bishop, D. V. M. (2014). Ten questions about terminology for children with unexplained language problems. International Journal of Language and Communication Disorders, 49(4), 381-415. https://doi.org/10.1111/1460-6984.12101

Bishop, D. V. M., Snowling, M. J., Thompson, P. A., \& Greenhalgh, T. (2017). Phase 2 
of CATALISE: A multinational and multidisciplinary Delphi consensus study of problems with language development: Terminology. Journal of Child Psychology and Psychiatry and Allied Disciplines. https://doi.org/10.1111/jcpp.12721

Bishop, D. V. M., Snowling, M. J., Thompson, P. A., Greenhalgh, T., Adams, C., Archibald, L., ... Whitehouse, A. (2016). CATALISE: A multinational and multidisciplinary Delphi consensus study. Identifying language impairments in children. PLoS ONE. https://doi.org/10.1371/journal.pone.0158753

Bishop, D. V. (1994). Is specific language impairment a valid diagnostic category? Genetic and psycholinguistic evidence. Philosophical Transactions of the Royal Society of London. Series B, Biological Sciences, 346(1315), 105-111. https://doi.org/10.1098/rstb.1994.0134

Conti-Ramsden, G., Knox, E., Botting, N., \& Simkin, Z. (2002). Educational placements and National Curriculum Key Stage 2 test outcomes of children with a history of specific language impairment. British Journal of Special Education, 29(2), 76-82. https://doi.org/10.1111/1467-8527.00244

Cotter, J. A. (2001). RightStart mathematics. Kindergarten lessons. Activities for Learning: Hazelton, ND.

Cowan, R., Donlan, C., Newton, E., \& Lloyd, D. (2005). Number skills and knowledge in children with specific language impairment. Journal of Educational Psychology, 97(4), 732-744. https://doi.org/10.1037/0022-0663.97.4.732

Dehaene, S. (1992). Varieties of numerical abilities. Cognition, 44(1-2), 1-42. https://doi.org/10.1016/0010-0277(92)90049-N

Dehaene, S., \& Cohen, L. (1995). Towards an anatomical and functional model of 
number processing. Mathematical Cognition. https://doi.org/10.1155/2014/461647

Dehaene, S., \& Cohen, L. (1995). Towards an anatomical and functional model of number processing. Mathematical Cognition.

Dockrell, J. E., Lindsay, G., \& Palikara, O. (2011). Explaining the academic achievement at school leaving for pupils with a history of language impairment: previous academic achievement and literacy skills. Child Language Teaching and Therapy, 27(2), 223-237. https://doi.org/10.1177/0265659011398671

Dockrell, J., \& Lindsay, G. (2000). Meeting the needs of children with specific speech and language difficulties. European Journal of Special Needs Education, 15(May), 24-41. https://doi.org/10.1080/088562500361682

Donlan, C. (1993). Basic numeracy in children with specific language impairment. Child Language Teaching and Therapy, 9(2), 95-104. https://doi.org/http://dx.doi.org/10.1177/026565909300900201

Donlan, C., Bishop, D. V, \& Hitch, G. J. (1998). Magnitude comparisons by children with specific language impairments: evidence of unimpaired symbolic processing. International Journal of Language \& Communication Disorders / Royal College of Speech \& Language Therapists, 33(2), 149-160. https://doi.org/10.1080/136828298247802

Donlan, C., Cowan, R., Newton, E. J., \& Lloyd, D. (2007). The role of language in mathematical development: Evidence from children with specific language impairments. Cognition, 103(1), 23-33.

https://doi.org/10.1016/j.cognition.2006.02.007

Donlan, C., \& Gourlay, S. (1999). The importance of non-verbal skills in the acquisition 
of place-value knowledge: Evidence from normally-developing and languageimpaired children. British Journal of Developmental Psychology, 17(1), 1-19. https://doi.org/10.1348/026151099165113

Durkin, K., Mok, P. L. H., \& Conti-Ramsden, G. (2015). Core subjects at the end of primary school: Identifying and explaining relative strengths of children with specific language impairment (SLI). International Journal of Language and Communication Disorders, 50(2), 226-240. https://doi.org/10.1111/14606984.12137

Durkin, K., Pearl, P. L., \& Conti-Ramsden, G. (2013). Severity of specific language impairment predicts delayed development in number skills. Frontiers in Psychology, 4(SEP). https://doi.org/10.3389/fpsyg.2013.00581

Fazio, B. B. (1994). The counting abilities of children with specific language impairment: a comparison of oral and gestural tasks. Journal of Speech and Hearing Research, $37(2), 358-368$.

Fazio, B. B. (1996). Mathematical abilities of children with specific language impairment: a 2-year follow-up. Journal of Speech and Hearing Research, 39(4), 839-849. https://doi.org/10.1044/jshr.3904.839

Fazio, B. B. (1999). Arithmetic calculation, short-term memory, and language performance in children with specific language impairment: a 5-year follow-up. Journal of Speech, Language, and Hearing Research, 42(2), 420-431. Retrieved from http://ezproxy.nottingham.ac.uk/login?url=http://search.proquest.com/docview/5751 9538?accountid=8018\%5Cnhttp://sfx.nottingham.ac.uk/sfx_local/?url_ver=Z39.88- 
2004\&rft_val_fmt=info:ofi/fmt:kev:mtx:journal\&genre=article\&sid=ProQ:ProQ:ass ia\&atitle $=$ Arithmetic

Gathercole, S. E., \& Baddeley, A. D. (1990). Phonological memory deficits in language disordered children: Is there a causal connection? Journal of Memory and Language, 29(3), 336-360. https://doi.org/10.1016/0749-596X(90)90004-J

Hall, N. E., \& Segarra, V. R. (2007). Predicting academic performance in children with language impairment: The role of parent report. Journal of Communication Disorders, 40(1), 82-95. https://doi.org/10.1016/j.jcomdis.2006.06.001

Harrison, L. J., McLeod, S., Berthelsen, D., \& Walker, S. (2009). Literacy, numeracy, and learning in school-aged children identified as having speech and language impairment in early childhood. International Journal of Speech-Language Pathology, 11(5), 392-403. https://doi.org/10.1080/17549500903093749

Joanisse, M. F., \& Seidenberg, M. S. (2003). Phonology and syntax in specific language impairment: Evidence from a connectionist model. Brain and Language, 86(1), 4056. https://doi.org/10.1016/S0093-934X(02)00533-3

Jordan, N. C., Levine, S. C., \& Huttenlocher, J. (1995). Calculation abilities in young children with different patterns of cognitive functioning. Journal of Learning Disabilities, 28(1), 53-64. https://doi.org/10.1177/002221949502800109

Kail, R. (1991). Developmental change in speed of processing during childhood and adolescence. Psychological Bulletin, 109(3), 490-501. https://doi.org/10.1037/00332909.109.3.490

Kleemans, T., Segers, E., \& Verhoeven, L. (2011). Precursors to numeracy in kindergartners with specific language impairment. Research in Developmental 
Disabilities, 32(6), 2901-2908. https://doi.org/10.1016/j.ridd.2011.05.013

Kleemans, T., Segers, E., \& Verhoeven, L. (2012). Naming speed as a clinical marker in predicting basic calculation skills in children with specific language impairment. Research in Developmental Disabilities, 33(3), 882-889. https://doi.org/10.1016/j.ridd.2011.12.007

Kleemans, T., Segers, E., \& Verhoeven, L. (2013). Relations between home numeracy experiences and basic calculation skills of children with and without specific language impairment. Early Childhood Research Quarterly, 28(2), 415-423. https://doi.org/10.1016/j.ecresq.2012.10.004

Koponen, T., Mononen, R., Räsänen, P., \& Ahonen, T. (2006). Basic numeracy in children with specific language impairment: heterogeneity and connections to language. Journal of Speech, Language, and Hearing Research : JSLHR, 49(1), 5873. https://doi.org/10.1044/1092-4388(2006/005)

Mainela-Arnold, E., Alibali, M. W., Ryan, K., \& Evans, J. L. (2011). Knowledge of mathematical equivalence in children with specific language impairment: insights from gesture and speech. Language, Speech, and Hearing Services in Schools, 42(January), 18-30. https://doi.org/10.1044/0161-1461(2010/09-0070)

Miller, C. A., Kail, R., Leonard, L. B., \& Tomblin, J. B. (2001). Speed of processing in children with specific language impairment. Journal of Speech Language and Hearing Research, 44, 416-433. https://doi.org/10.1044/1092-4388(2001/034) Mononen, R., Aunio, P., \& Koponen, T. (2014). A pilot study of the effects of RightStart instruction on early numeracy skills of children with specific language impairment. Research in Developmental Disabilities, 35(5), 999-1014. 
https://doi.org/10.1016/j.ridd.2014.02.004

Nelson, K. E., Welsh, J. A., Trup, E. M. V., \& Greenberg, M. T. (2011). Language delays of impoverished preschool children in relation to early academic and emotion recognition skills. First Language, 31(2), 164-194. https://doi.org/10.1177/0142723710391887

Nys, J., Content, A., Leybaert, J., \& Oetting, J. (2013). Impact of Language Abilities on Exact and Approximate Number Skills Development: Evidence From Children With Specific Language Impairment. Journal of Speech, Language \& Hearing Research, 56(3), 956-970. https://doi.org/10.1044/1092-4388(2012/10-0229)

Rice, M. L. (2003). A unified model of specific and general language delay: Grammatical tense as a clinical marker of unexpected variation. In Language competence across populations: Toward a definition of specific language impairment. (pp. 63-95).

Stone, C. A., \& Connell, P. J. (1993). Induction of a visual symbolic rule in children with specific language impairment. Journal of Speech and Hearing Research, 36, 599608.

Stothard, S. E., Snowling, M. J., Bishop, D. V. M., Chipchase, B. B., \& Kaplan, C. A. (1998). Stothard et al.: Language Impairment in Adolescence. JSLHR American Speech-Language-Hearing Association Journal of Speech, Language, and Hearing Research, 4198(407), 407-418.

Ullman, M. T., \& Pierpont, E. I. (2005). Specific Language Impairment is not Specific to Language: the Procedural Deficit Hypothesis. Cortex, 41(3), 399-433. https://doi.org/10.1016/S0010-9452(08)70276-4

Van Der Lely, H. K. J. (2005). Domain-specific cognitive systems: Insight from 
Grammatical-SLI. Trends in Cognitive Sciences.

https://doi.org/10.1016/j.tics.2004.12.002

Wexler, K., Schütze, C. T., \& Rice, M. (1998). Subject Case in Children With SLI and Unaffected Controls: Evidence for the Agr/Tns Omission Model. Language Acquisition, 7(2-4), 317-344. https://doi.org/10.1207/s15327817la0702-4_8

Willinger, U., Schmoeger, M., Deckert, M., Eisenwort, B., Loader, B., Hofmair, A., \& Auff, E. (2017). Screening for Specific Language Impairment in Preschool Children: Evaluating a Screening Procedure Including the Token Test. Journal of Psycholinguistic Research, pp. 1-11. https://doi.org/10.1007/s10936-017-9493-z 